\title{
Metabolic engineering of micronutrients in crop plants
}

\author{
Dieter Blancquaert, ${ }^{1}$ Hans De Steur, ${ }^{2}$ Xavier Gellynck, ${ }^{2}$ and Dominique Van Der Straeten ${ }^{1}$ \\ ${ }^{1}$ Laboratory of Functional Plant Biology, Department of Physiology, Ghent University, Ghent, Belgium. ${ }^{2}$ Division Agri-Food \\ Marketing \& Chain Management, Department of Agricultural Economics, Ghent University, Ghent, Belgium
}

Address for correspondence: Dominique Van Der Straeten, Laboratory of Functional Plant Biology, Department of Physiology, Ghent University, K.L. Ledeganckstraat 35, Ghent 9000, Belgium. dominique.vanderstraeten@ugent.be

\begin{abstract}
Micronutrient deficiency is a widespread phenomenon, most prevalent in developing countries. Being causally linked to the occurrence of a range of diseases, it affects billions of people worldwide. Enhancing the content of micronutrients in crop products through biotechnology is a promising technique to fight micronutrient malnutrition worldwide. Micronutrient fortification of food products has been implemented in a number of Western countries, but remains inaccessible for poor rural populations in a major part of the developing world. Moreover, evidence of the negative impacts of this practice on human health, at least for some vitamins, is accumulating. Biofortification of crop plantsthe enhancement of vitamins and minerals through plant biotechnology-is a promising alternative or complement in the battle against micronutrient deficiencies. Owing to a growing knowledge about vitamin metabolism, as well as mineral uptake and reallocation in plants, it is today possible to enhance micronutrient levels in crop plants, offering a sustainable solution to populations with a suboptimal micronutrient intake.
\end{abstract}

Keywords: metabolic engineering; biofortification; micronutrients; folate; iron; vitamin A

\section{Introduction}

The human body depends on micronutrients to sustain all physiological processes necessary to ensure good physical and mental health. Humans cannot produce these molecules themselves, and are therefore entirely dependent on their diet as a micronutrient source. Micronutrient deficiency, caused by the consumption of monotonous diets or micronutrient malabsorption, affects billions of people worldwide. ${ }^{1}$ Although this so-called "hidden hunger" is prevalent in Western countries, it mainly affects poor, rural populations in developing regions of the world. Even though staple crops provide a good source for the daily caloric intake, their micronutrient levels are usually low. Additionally, food storage, processing, and preparation cause substantial losses of micronutrients. In rice, for example, most of the micronutrients are concentrated in the outer layers of the kernel, the aleurone layer, which is removed upon milling to avoid rancidification. Rice milling is a widespread practice to prolong rice shelf life, but it lowers the nutritional value. The highest trace element deficiencies are found for iron, zinc, and iodine, each affecting nearly two billion people worldwide. Iron deficiency is the most prevalent nutritional disorder in the world and the only micronutrient deficiency that occurs significantly in developed countries as well. It is estimated that approximately one-third of the world's population suffers from anemia, which affects mostly children and women (http://www.who.int/ vmnis/database/anaemia/en/). Moreover, in developing countries, it is exacerbated by several infectious diseases, such as HIV, tuberculosis, and malaria. Insufficient iron uptake compromises a good pregnancy outcome, hampers normal physical and cognitive development, increases the risk of premature morbidity, and has an important economic effect through the reduction of work capacity in both individuals and populations (http://www. who.int/nutrition/topics/ida/en/). The most prevalent vitamin deficiencies are those of vitamin $\mathrm{A}$, vitamin $B_{9}$ (folate), and vitamin $B_{12}$ (cobalamin). Vitamin A deficiency (VAD) affects nearly 190 million preschool children and 19 million pregnant women, mostly in developing countries. ${ }^{2}$ VAD is 
the main cause of night blindness (nyctalopia), dry eyes (xerophthalmia), keratomalacia, and complete blindness. ${ }^{3}$ Folate deficiency is the main cause of neural tube defects (NTDs), and NTDS are used as a proxy to determine the folate status in a population. Annually, approximately 300,000 NTDs occur, mostly in Asian regions where rice is the main component of the diet. ${ }^{4}$ However, NTD prevalence alone underestimates the real occurrence of folate deficiency, which is estimated to affect billions of people worldwide, giving it epidemic proportions. ${ }^{5}$

Several strategies can be implemented to fight micronutrient deficiencies, each with its own drawbacks and limitations (extensively reviewed in Ref. 5). Dietary diversification is the most obvious way to address micronutrient malnutrition. However, this strategy requires recurrent educational efforts and a change in dietary habits. In several countries, industrial fortification with micronutrients of food products, such as flour, breakfast cereals, oils, and milk, is mandatory. Although this practice is proven to be successful in most cases, policy makers need to be sure that this strategy is safe and that supraoptimal intake of micronutrients, which sometimes can have adverse effects on human health, is avoided. Owing to the fact that most people are unaware of their micronutrient status, supplementation in the form of pills or injections often does not have the desired outcome. Moreover, the supplementation strategy requires educational efforts and often does not reach the target group of a population. In addition, micronutrient fortification and supplementation requires specialized infrastructure, which is difficult to implement in developing countries, where micronutrient deficiency is the highest. A biofortification strategy enhancing the natural micronutrient levels in crops is a valuable solution to address hidden hunger, especially in poor, rural populations. Biofortification can be achieved in two ways: conventional breeding and/or metabolic engineering. Conventional breeding relies on the natural variation of the target micronutrient levels within sexually compatible individuals of a crop. In most cases, this variation is low and insufficient to reach target micronutrient levels. ${ }^{6,7}$ Moreover, conventional breeding is time-consuming, requiring several rounds of crossing and screening, although these processes can be accelerated with the aid of quantitative trait loci mapping and marker-assisted breeding. Metabolic engineering has proven to be a successful, sustainable, and cost-effective strategy in crop biofortification. ${ }^{7,8}$ In this review, we shed light on different aspects of metabolic engineering and demonstrate, through case studies, that this strategy can become a valuable player to address micronutrient deficiencies globally. In addition, vitamin bioavailability and examples of tackling vitamin stability crop products are discussed.

\section{Crop biofortification through metabolic engineering}

Metabolic engineering can be used to enhance nutritional value in crop plants by engineering metabolic pathways through genetic modification. This strategy involves the modulation of endogenous metabolic pathways or the introduction of one or more heterologous actors to (1) enhance the production of a target compound, (2) reduce the levels of an undesirable molecule, or (3) modulate the flux to induce the accumulation of a more bioavailable, stable, and active compound (reviewed in Ref. 8). Metabolic engineering requires a thorough knowledge of the regulation of the endogenous metabolic pathways involved. Only then can a successful engineering strategy be developed in which key enzymes are targeted for overexpression or downregulation to enhance the biosynthesis or accumulation of micronutrients without affecting crop development and yield. Depending on the source of the inserted gene(s), engineered crops can be classified as transgenic, intragenic, or cisgenic. Transgenic crops contain transgenes from sexually incompatible organisms, whereas intragenic crops are created by using genes from closely related (and sexually compatible) individuals, allowing in vitro recombination of elements of different genes within the sexually compatible gene pool. In cisgenic crops, however, this recombination is not allowed and the inserted gene needs to contain its original organization in the normal orientation. Through the use of tissue-specific promoters driving the expression of targeted (trans)genes, metabolic engineering enables the accumulation of micronutrients in tissues where they do not occur naturally or accumulate only in very low amounts. In these specific cases, conventional breeding is of very little or no use, and metabolic engineering is the only way forward. The different aspects of metabolic engineering are illustrated in the following case studies. In each example, we emphasize the most important 
candidate enzymes for engineering and the results obtained thus far, together with perspectives and suggestions to further increase the nutritional value of crops in future biofortification studies.

\section{Vitamin A}

Vitamin A is important in many aspects of human development and growth. It is involved in epithelial differentiation, reproduction, proper immune function, and vision. ${ }^{9}$ VAD can eventually lead to permanent blindness and is able to enhance the occurrence and severity of infectious diseases. ${ }^{10}$ The recommended dietary allowance (RDA) is $900 \mu \mathrm{g}$ for men and $700 \mu \mathrm{g}$ for women of retinol activity equivalents per day (National Institutes of Health, Office of Dietary Supplements). Retinoids (vitamin A) are a group of molecules with a retinyl group as a common skeleton. This retinyl group is composed of a $\beta$-ionone ring and an isoprenoid side chain. Meat and dairy products contain retinyl esters, which are converted to retinol in the small intestine. Plants, however, contain carotenoids as a source of provitamin $A$, of which $\beta$-carotene has the highest provitamin A activity. Upon ingestion, provitamin A and retinol are converted to retinal and retinoic acid, the functional vitamin A molecules. Plants produce four provitamin A carotenoids (PACs) $(\alpha-, \beta$-, and $\gamma$-carotene and $\beta$ cryptoxanthine), each containing at least one retinyl group. Carotenoids are tetraterpenoids with a C40 linear backbone. Provitamin A biosynthesis (Fig. 1) starts with the condensation of two geranylgeranyl pyrophosphate (GGPP) molecules to form 15cis-phytoene by phytoene synthase (PSY, encoded by $\operatorname{crtB}$ in bacteria). ${ }^{11}$ GGPP is the condensation product of three isopentenyl diphosphate molecules and one dimethylallyldiphosphate, a reaction performed by GGPP synthase (or the bacterial equivalent CRTE) in the plastids. ${ }^{12}$ To produce the carotenoid chromophore, four desaturation reactions are performed. In plants, these reactions are the result of phytoene desaturase and $\zeta$-carotene desaturase. Subsequently, all-trans-lycopene is produced by carotenoid isomerase in nongreen tissue and by light and chlorophyll in green tissue. ${ }^{13-15}$ In bacteria, 15-cis-phytoene is directly converted to all-trans-lycopene by CRT1. All-trans-lycopene acts as a substrate of two competing cyclases: lycopene $\varepsilon$-cyclase (LYCE) and lycopene $\beta$-cyclase (LYCB; CRTY). LYCB adds a $\beta$-ring to lycopene, gener- ating $\gamma$-carotene. A second addition of a $\beta$-ring by LYCB results in the formation of $\beta$-carotene. $\gamma$-Carotene does not accumulate and is rapidly converted to $\beta$-carotene. ${ }^{16,17}$ The action of LYCE produces $\delta$-carotene, which has no provitamin A activity. LYCB can also use $\delta$-carotene to add a $\beta$-ring to the molecule, generating $\alpha$-carotene, which has provitamin A activity. $\alpha$-Carotene and $\beta$-carotene can be further converted to lutein (via zeinoxanthin or $\alpha$-cryptoxanthin) and zeaxanthin (via $\beta$-cryptoxanthin), respectively, by the action of $\beta$-carotene hydroxylase (CRTZ in bacteria). ${ }^{18}$ Although these pigments have no provitamin A activity, they are considered to be powerful antioxidants.

The first transgenic crop biofortified with provitamin A was rice. Rice endosperm does not contain provitamin A naturally; therefore, metabolic engineering was the only way forward to enhance provitamin A content. Owing to the yellow color of the transgenic rice kernels, it was termed "golden rice." Initially, transgenes from daffodil ( $p s y$ and $l y c b$ ) and the bacterium Pantoea ananatis (formerly known as Erwinia uredovora) (PacrtI) were used in the first-generation golden rice. ${ }^{19}$ An accumulation of total carotenoids up to $1.6 \mu \mathrm{g} / \mathrm{g}$ dry weight was reported. ${ }^{19}$ In second-generation golden rice (golden rice 2), the daffodil gene psy was replaced with $p s y 1$ from corn, which, in combination with PacrtI, further raised $\beta$-carotene content to approximately $30 \mu \mathrm{g} / \mathrm{g}$ dry weight. ${ }^{20}$ The creation of golden rice 1 and 2 opened the door for the development of other "golden" crops, such as potato, corn, wheat, cassava, and sorghum. In corn and wheat, the same genes were applied as in golden rice $2 .{ }^{21,22}$ Overexpression of Arabidopsis 1-deoxyxylulose-5phosphate synthase (AtDXS), a protein important in the flux toward GGPP, in combination with PacrtB on the one hand and corn psyl and PacrtI on the other hand, enhanced $\beta$-carotene content in cassava and sorghum, respectively. ${ }^{23,24}$ In potato, the best results were obtained with the use of $\operatorname{crtB}, \operatorname{crtI}$, and $c r t Y$ transgenes, all from P. ananatis. ${ }^{25}$

\section{Vitamin $E$}

Vitamin $\mathrm{E}$ is a lipophilic compound that acts as a powerful antioxidant in the prevention of a range of diseases caused by oxidative degeneration. ${ }^{26}$ The RDA of vitamin E is $15 \mathrm{mg}$ per day for adults and $7 \mathrm{mg}$ for children up to 8 years (National Institutes 


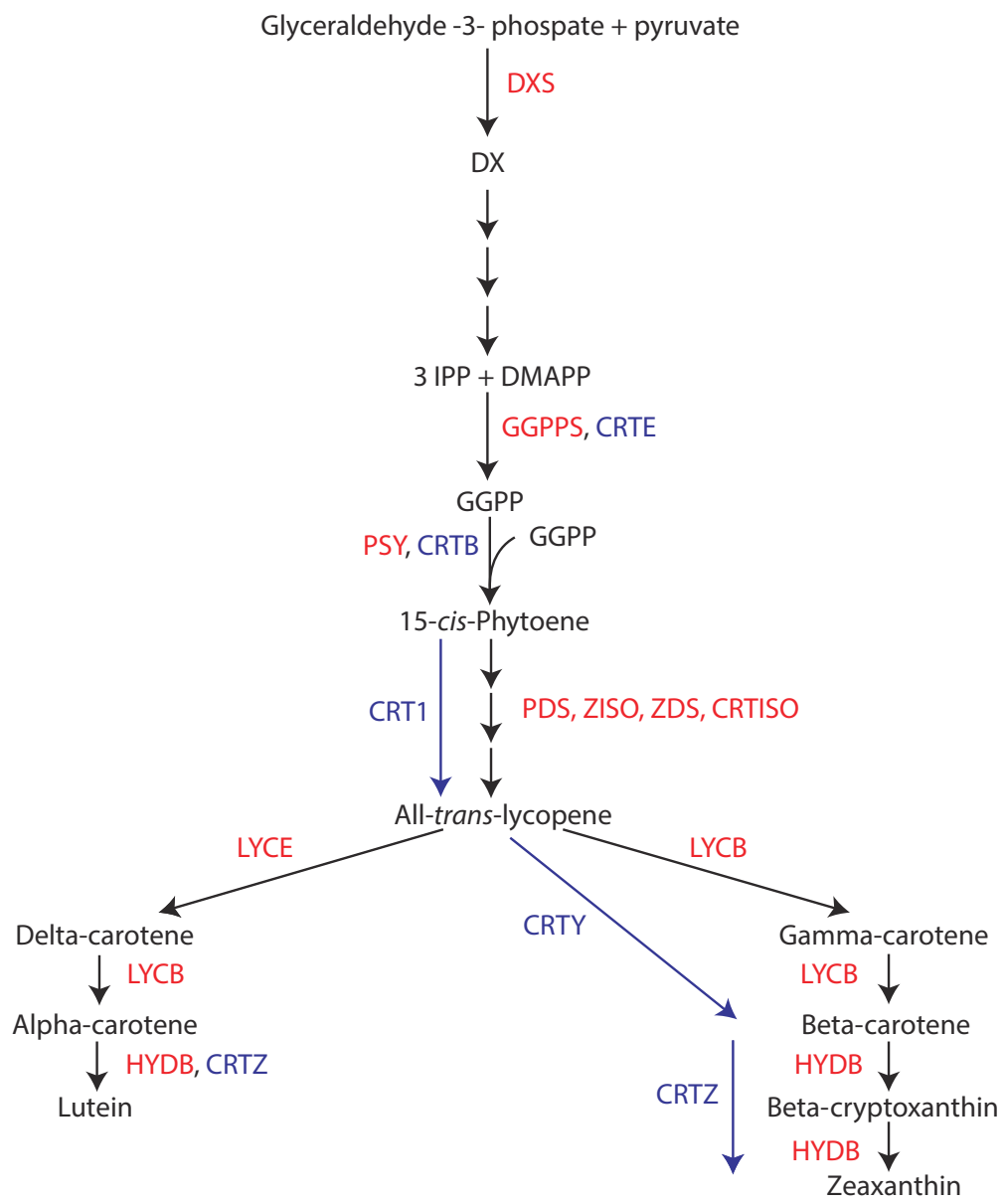

Figure 1. Schematic and simplified representation of carotenoid biosynthesis in plants. Only $\boldsymbol{\alpha}$-carotene, $\boldsymbol{\beta}$-carotene, and $\boldsymbol{\beta}$ cryptoxanthin are important contributors to provitamin A activity. Plant enzymes are depicted in red, their bacterial counterparts in blue. CRTB, bacterial phytoene synthase; CRT1, bacterial phytoene desaturase; CRTE, bacterial GGPPS; CRTISO, carotenoid isomerase; CRTY, bacterial lycopene $\boldsymbol{\beta}$-cyclase; CRTZ, bacterial $\boldsymbol{\beta}$-carotene hydroxylase; DMAPP, dimethylallyldiphosphate; DX, 1deoxyxylulose-5-phosphate; DXS, DX synthase; GGPP, geranylgeranyl pyrophosphate; GGPPS, GGPP synthase; HYDB, $\boldsymbol{\beta}$-carotene hydroxylase; IPP, isopentenyl diphosphate; LYCB, lycopene $\boldsymbol{\beta}$-cyclase; LYCE, lycopene $\boldsymbol{\varepsilon}$-cyclase; PDS, phytoene desaturase; PSY, phytoene synthase; ZDS, $\zeta$-carotene desaturase; ZISO, $\zeta$-carotene isomerase.

of Health, Office of Dietary Supplements). Tocochromanols is the collective term for eight species of tocopherols and tocotrienols, differing in the position and number of methyl units ( $\alpha$ : three methyl units; $\beta$ : two methyl units; $\gamma$ : two methyl units; $\delta$ : one methyl unit). However, these lipid-soluble molecules have different levels of vitamin E activity, the highest activity being found in $\alpha$-tocopherol. Oilseeds have high total tocopherol levels, although most of it is $\gamma$-tocopherol. ${ }^{27}$ The biosynthesis pathway of tocopherol is well understood and involves five VTE enzymes (VTE 1-5). The pathway starts with hydroxyphenylpyruvate, which is converted to homogentisate (HGA) by hydroxyphenylpyruvate dioxygenase (HPPD). Upon HGA biosynthesis, tocochromanol production branches toward the production of tocotrienols and tocopherols. In the tocopherol branch, phytol kinase (VTE5) phosphorylates phytol-to-phytyl monophosphate, which is again phosphorylated by a kinase to form phytyldiphosphate (PDP). PDP and HGA are condensed to 2-methyl-6-phytyl-1,4-benzoquinol (MPBQ) by homogentisate phytyltransferase (VTE2; HPT)). A second methyl group can be attached to the MPBQ molecule by MPBQ methyltransferase (VTE3; MPBQ-MT) to obtain 2,3-dimethyl-6-phytyl-1, 
4-benzoquinol (DMPBQ). A cyclization reaction, performed by tocopherol cyclase (VTE1), produces $\gamma$-tocopherol and $\delta$-tocopherol from MPBQ and DMPBQ, respectively. A second (and third) methylation reaction is catalyzed by $\gamma$-tocopherol methyltransferase (VTE4; $\gamma$-TMT) to obtain $\beta$ tocopherol from $\delta$-tocopherol and $\alpha$-tocopherol from $\gamma$-tocopherol. Similar reactions with the same enzymes are involved in the tocotrienol branch, but geranylgeranyl pyrophosphate (GGPP) is used together with HGA by homogentisate geranylgeranyl transferase (HGGT) to produce 2-methyl-6geranylgeranyl-plastoquinol, the tocotrienol equivalent of MPBQ. Several engineering attempts have been undertaken to (1) increase tocochromanol content in crops and (2) shift the accumulation of tocochromanols in favor of $\alpha$-tocochromanols. In Arabidopsis, soybean, and canola seeds, overexpression of a bacterial prephenate dehydrogenase, which catalyzes the conversion of prephenate to hydroxyphenylpyruvate, in combination with AtHPPD, resulted in a fourfold increase in total tocochromanol levels. ${ }^{28}$ The authors clearly showed that these first steps in tocochromanol biosynthesis are indeed rate limiting. In rice seeds, although overexpression of AtHPPD resulted in a marginal increase in tocochromanol levels and no increase in total tocopherol content, it caused a shift from $\gamma$ - to $\alpha$-tocopherol. ${ }^{29}$ In corn, overexpression of barley HGGT increased total tocochromanol content by sixfold. ${ }^{30}$ Constitutive overexpression of AtHPPD and AtVTE3 tripled the amount of $\gamma$-tocopherol in corn seeds. ${ }^{31}$ In rice, constitutive and endospermspecific overexpression of AtTMT did not change total tocopherol and tocotrienol levels but greatly enhanced $\alpha$-tocotrienol levels, at the expense of $\gamma$ and $\delta$ - tocotrienols. ${ }^{32}$ Altogether, it becomes clear that, to successfully enhance vitamin E content in crop plants, a multitarget approach is required, boosting the production of tocopherol precursors HGA and PDP and diverting the flux toward $\alpha$ tocopherol production.

\section{Vitamin $B_{6}$}

Vitamin $B_{6}$ reduces the risk of diabetes, cardiovascular disease, hypertension, kidney disease, neurological disorders, pellagra, and epilepsy. ${ }^{33}$ The RDA is $1.3 \mathrm{mg}$ per day for adults (National Institutes of Health, Office of Dietary Supplements). Vitamin $B_{6}$ comprises six vitamers, including pyridox- ine, pyridoxal, and pyridoxamine and its phosphorylated derivatives. Pyridoxal-5-phosphate is considered to be the most important vitamer, since it acts as a cofactor in more than 140 chemical reactions in the cell. ${ }^{33}$ Therefore, the accumulation of this vitamer is the primary goal in a biofortification strategy. Cross talk between plant vitamin $\mathrm{B}_{6}$ biosynthesis and salvage pathways exists, which enables them to interconvert among the different vitamers. ${ }^{34}$ Most of the key players in vitamin $\mathrm{B}_{6}$ biosynthesis and regulation have been identified, knowledge that is important from a metabolic engineering perspective. The de novo biosynthesis of vitamin $B_{6}$ in plants is mediated by only two enzymes, ${ }^{35}$ PDX1 and PDX2, in a pathway that is independent from the deoxyxylulose-5-phosphate (DXP) pathway found in some bacteria. ${ }^{36,37}$ The Arabidopsis,${ }^{35}$ rice, $^{38}$ and cassava ${ }^{39}$ genomes have three homologues of PDX1 (PDX1.1, PDX1.2, and $P D X 1.3)$ and one PDX2 gene. Only PDX1.1 and PDX1.3 are considered to be important in vitamin $\mathrm{B}_{6}$ biosynthesis. ${ }^{35}$ In tobacco, overexpression of PDX1 and PDX2 from Cercospora nicotianae, a plant fungal pathogen, resulted in a modest $20 \%$ increase in vitamin $\mathrm{B}_{6}$ content. ${ }^{40}$ However, this coincided with a delay in seed germination and plant growth. ${ }^{40}$ In Arabidopsis, constitutive overexpression of AtPDX1.3 and AtPDX2 again raised vitamin $\mathrm{B}_{6}$ content in seeds by $20 \%$, whereas seedspecific expression resulted in a threefold increase in seeds ${ }^{41}$ without affecting plant performance. Constitutive overexpression of another PDX1 homologue, AtPDX1.1, in combination with AtPDX2 further increased vitamin $\mathrm{B}_{6}$ content, and pyridoxal-5-phosphate in particular, by fivefold in Arabidopsis, clearly indicating that the correct choice of homologues is important. ${ }^{42}$ Interestingly, this biofortification led to an increase in seed size through embryo enlargement and larger aerial organs. ${ }^{42}$ Recently, constitutive and root-specific overexpression of AtPDX1.1 and AtPDX2 in cassava resulted in several-fold higher vitamin $\mathrm{B}_{6}$ levels in leaves and roots, of which the highest increase could be detected for the unphosphorylated vitamers. ${ }^{43}$

\section{Vitamin $B_{9}$}

Vitamin $B_{9}$ is the collective term of a group of water-soluble B vitamins-folates-that play important roles as cofactors in one-carbon $(\mathrm{C} 1)$ metabolism in all living organisms. ${ }^{6}$ In several 
metabolic processes, from methylation to DNA synthesis, they provide or accept $\mathrm{C} 1$ units in the form of methyl, methenyl, methylene, or formyl groups. The molecule backbone consists of three parts: a pterin group, a para-aminobenzoate ( $p$ ABA) moiety, and a glutamate tail. Folates differ in the $\mathrm{C} 1$ unit attached to the molecule and the length of the glutamate tail, comprising tetrahydrofolate (THF), the most reduced form, and its derivatives 5-methyl THF, 5-formyl THF, 10-formyl THF, and 5,10-methylene THF, which all can exist as monoglutamates or polyglutamates. Each of these molecules, without considering the length of the glutamate tail, has specific functions in $\mathrm{C} 1$ metabolism. ${ }^{44}$ The RDA of folates is $400 \mu \mathrm{g}$ for adults and $600 \mu \mathrm{g}$ for pregnant women (National Institutes of Health, Office of Dietary Supplements). Folate deficiency may cause NTDs, such as cleft spine ${ }^{45}$ and megaloblastic anemia. ${ }^{46}$ Moreover, insufficient folate intake increases the risk of cardiovascular diseases, ${ }^{47}$ coronary diseases, ${ }^{48}$ stroke, ${ }^{49}$ Alzheimer's disease, ${ }^{50}$ and a range of cancers. ${ }^{51}$ Currently, synthetic folic acid (FA), the fully oxidized form, is used in fortification and supplementation programs. However, FA needs to be reduced to dihydrofolate (DHF) and THF to become active, and excessive FA intake can have adverse effects on human health. ${ }^{5}$ Folate biosynthesis (Fig. 2) involves three subcellular compartments: $p$-ABA is synthesized in the plastids and pterin is synthesized in the cytosol, and both precursors are condensed, reduced, and glutamylated in the mitochondria. The first engineering attempts focused on two key enzymes in the folate biosynthesis pathway: GTP cyclohydrolase I (GTPCHI, G), the first step in the pterin branch, and aminodeoxychorismate synthase (ADCS, A), the first step in the $p$ ABA branch. Overexpression of GTPCHI or ADCS alone greatly enhanced pterin or $p$-ABA content, but caused a depletion of the nontargeted other precursor, with moderate increases in folate content obtained only in G lines of Arabidopsis, ${ }^{52,53}$ rice, ${ }^{54,55}$ tomato, ${ }^{56}$ lettuce, ${ }^{57}$ potato, ${ }^{53}$ and corn. ${ }^{21}$ Simultaneous overexpression of GTPCHI and ADCS by crossing $G$ and $A$ lines in tomato ${ }^{58}$ or by single-locus T-DNA insertion (GA lines) in rice ${ }^{54}$ resulted in a massive accumulation of folates in the engineered crops. In GA rice lines, folate levels up to $1723 \mu \mathrm{g} / \mathrm{g}$ fresh weight could be measured. ${ }^{54}$ However, this two-gene approach did not give the desired results in potato tubers or in Arabidopsis, pointing to another bottleneck in the folate biosynthesis pathway in these plants. ${ }^{53}$ Since pterin and p-ABA content is high in these plants, it was suggested that a triple-gene approach with the bifunctional enzyme hydroxymethyldihydropterin pyrophosphokinase/dihydropteroate synthase (HPPK/DHPS) may be more successful. ${ }^{5}$ Dong et al. evaluated the effect of GTPCHI overexpression, in combination with DHF synthetase (DHFS) or folylpolyglutamate synthetase (FPGS), on folate levels in rice seeds. ${ }^{55}$ As expected, only a moderate enhancement in folate content could be detected, due to a depletion of the $p$-ABA pool in the engineered lines. More recently, the combined overexpression of GTPCHI, ADCS, and a fusion between a synthetic folate-binding protein (FBP) and $\beta$-carbonic anhydrase 2 from Arabidopsis in rice resulted in an additional increase in folate levels of $50 \%$ as compared with the original GA lines, ${ }^{59}$ indicating that folate sequestration by this chimeric protein allows a higher accumulation of this vitamin in rice seeds.

\section{Iron}

Iron is an important component of numerous enzymes, including hemoglobin. Iron deficiency can result in anemia, causing 0.8 million deaths annually. ${ }^{60}$ The RDA of iron is $8 \mathrm{mg}$ per day for male adults and $18 \mathrm{mg}$ for women (National Institutes of Health, Office of Dietary Supplements). Of all the metal-uptake mechanisms in plants, iron acquisition is the best studied. This is mainly due to the clearly defined physiological aspects and consequences of iron deficiency in plants. From the rhizosphere, iron is absorbed through the root epidermis, transported to the xylem, and relocated throughout the plant to different tissues, where it can be stored upon further demand. For the sake of simplicity, only the main actors in these processes are mentioned next. Iron deficiency in plants is mainly caused by the low solubility of $\mathrm{Fe}$ (III), which is most prominent in alkaline and neutral soils, rather than a low iron content. Therefore, plants have developed two strategies to increase Fe(III) solubility (Fig. 3). Both strategies are promoted by low iron availability in the soil and considered to be high-affinity uptake systems. When sufficient iron is available, low-affinity transport systems are implemented. The latter are, however, still far from fully 


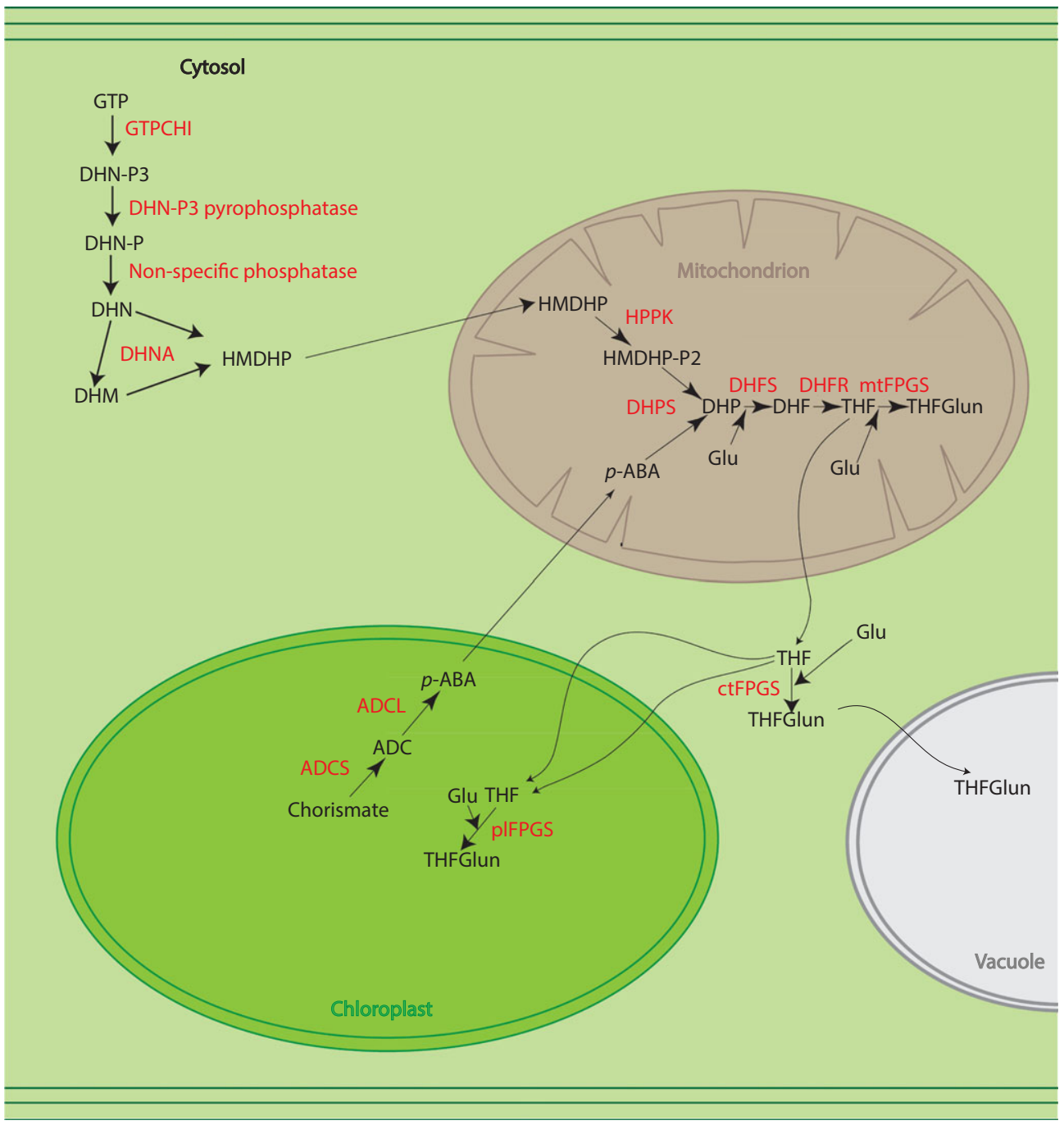

Figure 2. Schematic representation of the folate biosynthesis pathway in plants. Folate biosynthesis is characterized by a compartmentalization in the cytosol, the plastids, and the mitochondria. Enzymatic steps are indicated in red. ADC, aminodeoxychorismate; $\mathrm{ADCS}, \mathrm{ADC}$ synthase; $\mathrm{ADCL}, \mathrm{ADC}$ lyase; ctFPGS, cytosolic folylpolyglutamate synthetase; DHF, dihydrofolate; DHFR, DHF reductase; DHFS, DHF synthetase; DHM, dihydromonapterin; DHN, dihydroneopterin; DHNA, DHN aldolase; DHN-P, dihydroneopterin monophosphate; DHN-P3, dihydroneopterin triphosphate; DHP, dihydropteroate; DHPS, DHP synthase; Glu, glutamate; GTP, guanosine triphosphate; GTPCHI, GTP cyclohydrolase 1; HMDHP, 6-hydroxymethyldihydropterin; HMDHP-P2, 6-hydroxymethyldihydropterin pyrophosphate; HPPK, dihydropterin pyrophosphokinase; mtFPGS, mitochondrial FPGS; $p$-ABA, para-aminobenzoate; plFPGS, plastidial FPGS; THF, tetrahydrofolate; THFGlun, tetrahydrofolate polyglutamate.

elucidated and therefore currently not considered as targets for metabolic engineering. The first strategy (strategy I), characteristic for eudicots and nongraminaceous monocots, involves the acidification of the soil by $\mathrm{H}^{+}$-ATPases, pumping protons into the apoplast and rhizosphere, and the excretion of small chelating molecules, such as malate and citrate. In this way, $\mathrm{Fe}(\mathrm{III})$ is solubilized and chelated. Bound
$\mathrm{Fe}$ (III) is subsequently reduced at the root surface by plasma membrane-bound $\mathrm{Fe}$ (III) chelate reductases. In Arabidopsis, the ferric reductase oxidase (FRO) family consists of eight members, of which AtFRO2 has the highest iron reductase activity. ${ }^{61}$ Moreover, this gene is mainly expressed in the roots and therefore considered to be the main attributor of $\mathrm{Fe}(\mathrm{III})$ reduction in the rhizosphere. ${ }^{61}$ Rice 


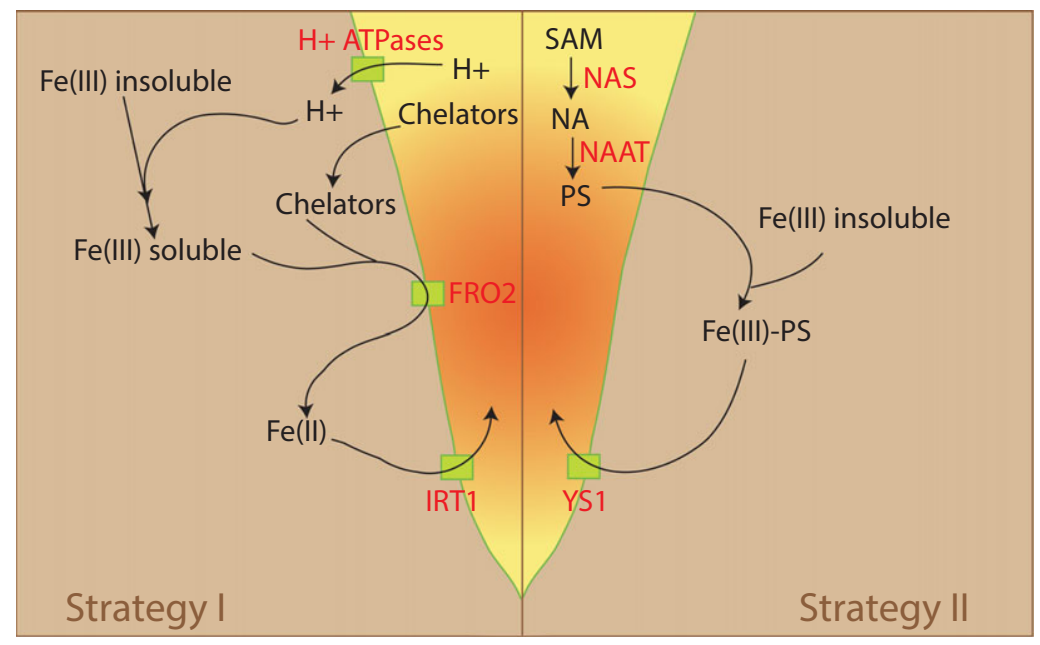

Figure 3. Schematic representation of iron uptake in plant roots and root hairs upon iron deficiency. Strategy I is applied by eudicots and nongraminaceous monocots. Strategy II is characteristic for grasses. Rice has features of both strategies. Enzymes are indicated in red. FRO2, ferric reductase oxidase 2; IRT1, iron-regulated transporter 1; NA, nicotianamine; NAAT, NA aminotransferase; NAS, NA synthase; PS, phytosiderophore; SAM, S-adenosyl methionine; YS1, yellow stripe 1.

transformed with AtFRO2 did not exhibit a high root reductase activity. ${ }^{62}$ However, in this attempt, the 0.6-kb upstream promoter region of this Arabidopsis gene was used instead of a rice promoter active in the root epidermis, and no transcript levels of the transgene could be detected. ${ }^{62}$ Constitutive overexpression of AtFRO2 in soybean resulted in fivefold higher iron concentrations in leaves and pod walls, but only a $10 \%$ increase of iron content could be detected in the seeds, suggesting that additional factors are required to translocate iron to the seed. ${ }^{63}$ Upon reduction, $\mathrm{Fe}(\mathrm{II})$ is transported into the root by IRT1 (iron-regulated transporter 1), a member of the ZIP (zinc-regulated transporter, IRT-like protein) family of metal transporters.

Strategy II plants (grasses) release phytosiderophores (PS) into the rhizosphere, which chelate $\mathrm{Fe}$ (III) before uptake. PS are derived from nicotianamine, a condensation product of three molecules of $S$-adenosyl methionine. Following nicotianamine production by nicotianamine synthase (NAS), deoxymugineic acid is formed by nicotianamine aminotransferase and subsequently converted to mugineic acid and its derivatives, which act as PS. The PS-Fe(III) complex is imported into the roots by yellow stripe 1 (YS1), which was first discovered in maize. Interestingly, Arabidopsis (strategy I) has eight homologues of this gene (yellow stripe-like (YSL)). Although strategy I plants do not produce PS, these proteins may play a role in the transport of metal-nicotianamine complexes in the plant. Nicotianamine is present in higher plants, acting as a general metal chelator. ${ }^{64}$ Rice combines both strategies, since $\mathrm{Fe}(\mathrm{II})$ is readily available in anaerobic waterlogged soils, and there is no need to solubilize Fe(III). Therefore, it produces less PS (strategy II) than barley and maize and expresses OsIRT1 and OsIRT2 (strategy I) genes to compensate. ${ }^{65}$

Since Fe is highly reactive, it is assumed that $\mathrm{Fe}$ is bound to chelators after uptake by the root epidermis. Symplastic transport brings iron through the root cortex to the pericycle, where it is loaded into the xylem for further transport throughout the plant. To prevent precipitation, Fe is chelated, most likely to citrate in the vasculature. ${ }^{65,66}$ Developing seeds acquire iron from the roots and senescent leaves via the phloem. Iron, upon complexation with nicotianamine, is translocated from the xylem to the phloem by YSL proteins. ${ }^{66}$ In rice, OsIRT1 is responsible for loading $\mathrm{Fe}(\mathrm{II})$ into the phloem, where it is likewise chelated to nicotianamine. Next to phloem loading, YSL proteins are also pivotal in the delivery of iron to the seeds. ${ }^{65}$ In developing Arabidopsis seeds, Fe is most likely bound to phytate (myo-inositol hexakisphosphate) and stored in the vacuoles, ${ }^{65}$ whereas only a small fraction of seed iron is bound to ferritin in the plastids. ${ }^{67}$ Phytate is the principal phosphorus storage form in the seed, 
accounting for approximately $65-80 \%$ of total seed phosphorus. ${ }^{68}$ In most cereals (with the exception of maize), phytate is mostly $(80 \%)$ concentrated in the outer layers of the seed (aleurone layers) and the embryo, where it forms phytate globoids in protein storage bodies. Phytate has the ability to chelate a number of divalent cations, such as iron, magnesium, and zinc, upon which a mixed salt, phytin, is formed. Since monogastric animals, such as humans, fish, and poultry, lack phytase, the enzyme responsible for phytate degradation (and the concomitant release of free phosphorus, metals, and myo-inositol), ${ }^{69}$ high phytate levels in food and feed lower their nutritional value. Hence, phytate is considered an antinutrient. Moreover, agronomic practice (by supplementation of animal feed with bioavailable phosphorus and by phytate discharge through excrements), contributes to water eutrophication. ${ }^{70}$ Therefore, lowering phytate levels in crop plants is beneficial for both humans and the environment. Phytate is omnipresent in eukaryotes and plays an essential role in many cellular functions. Several low phytic acid (lpa) mutants were identified in crop plants, including rice, wheat, sorghum, maize, and barley. However, although an increase in inorganic phosphorus levels was seen in lpa plants, it often coincided with changes in plant performance and crop yield. Therefore, seedspecific knockdown of phytate biosynthesis genes is a more suitable strategy to create low-phytate crops without yield penalties. Its biosynthesis starts with the production of the myo-inositol backbone, which is afterwards phosphorylated to form phytate. Seed-specific manipulation of the expression of myo-inositol 3-phosphate synthase, the first gene in phytate biosynthesis, in rice resulted in approximately $50 \%$ more free phosphorus than in $l p a$ mutants. ${ }^{71}$ However, myo-inositol is an important metabolite in many metabolic processes in plants, and disruption of its biosynthesis can result in unwanted side effects. Therefore, targeting enzymes downstream of the phytate biosynthesis pathway is more suitable. In this respect, seed-specific silencing of inositol phosphate kinase, the last step in the production of phytate, resulted in $69 \%$ reduction in phytate levels (and an increase in inorganic phosphate), together with an $80 \%$ increase of iron content in rice seeds. ${ }^{72}$ Through map-based cloning, an ATP-binding cassette transporter, a key player in phytate accumulation in soybean seed and maize, was identified at the lpa locus of mutations. ${ }^{73}$ This transporter could be a suitable candidate for engineering phytate levels, since it is not believed to play a role in other metabolic pathways. ${ }^{73}$ Alternatively, the overexpression of phytase in seeds could be considered. In this way, phytate in engineered crop and, after ingestion, in other feed products could be degraded. ${ }^{74}$

Next to lowering phytate levels, enhancing ferritin content in crop seeds holds great promise for enhancing iron content in seeds. Plant ferritins (and ferritins in general) consist of an apoferritin coat, the shell, and an iron core, which may contain up to 4500 iron atoms. ${ }^{75}$ They act as acceptors and donors of iron in metabolic processes and are considered to protect the plant against toxic free iron, rather than to be an iron-storage form. ${ }^{67}$ Moreover, ferritin-bound iron is highly bioavailable during digestion. Overexpression of ferritin in plants only resulted in moderate increases in iron seed content, owing to the fact that, in seeds, most iron is stored in vacuoles instead of plastids, where ferritin accumulates. ${ }^{76}$ Therefore, in order to be successful, a seed ferritin approach needs to be accompanied by a translocation of iron from the vacuoles to the plastids. In Arabidopsis, AtVIT1, AtNRAMP3, and AtNRAMP4 are shown to be important in vacuolar iron transport in the seeds. ${ }^{76}$ Impairment of vacuolar iron import concomitant with a promotion of accumulation of iron in the plastids is imperative to make a ferritin-based approach successful.

It is clear that crop biofortification with iron involves many processes, from iron uptake by the roots to iron storage in the edible parts. In order to be successful, these processes need to be correctly orchestrated, which is only possible in a multigene approach, with the use of tissue-specific promoters. The best results in rice so far were obtained via the manipulation of iron translocation (NAS) together with the promotion of iron accumulation in the endosperm (YSL2) and the enhancement of iron sequestration by ferritin, which increased rice seed iron content sixfold. ${ }^{77}$

\section{Metabolic engineering of vitamin stability in crop plants}

Vitamins are unstable molecules, sensitive to changes in temperature, $\mathrm{pH}$, and humidity, and they are prone to (photo)-oxidative degradation. ${ }^{78}$ Vitamin stability differs greatly among vitamers. In 
the vitamin $\mathrm{B}_{9}$ group, FA and 5-formyl THF are the most stable molecules, ${ }^{79}$ while THF and DHF are the most sensitive to degradation. ${ }^{6}$ The effect of food processing on vitamin stability has been well studied; however, only a few studies monitored the effect of storage time, although it is commonly known that vitamins degrade over time. In freeze-dried fortified apple, vitamin E levels dropped about 50\% after 6 months of storage, ${ }^{80}$ while only $11 \%$ of vitamin C content is left in guava nectar. ${ }^{81} \mathrm{In}$ biofortified corn, provitamin A content dropped approximately 70\% after half a year. ${ }^{82}$ In general, staple crops are stored until the next harvest. In rice-producing countries, the storage period is region dependent (depending on the number of growth seasons), ranging from several months to years before consumption. ${ }^{83}$ Corn, after drying, can be stored up until 9 months or longer. ${ }^{84}$ In these and other, mostly cereal crops, it is important that vitamin stability is ensured over time. Only then can the implementation of biofortified crops be valuable in the battle against micronutrient deficiencies. Unfortunately, the effect of storage time on vitamin stability is largely neglected in most biofortification studies. Recently, it was shown that folate levels in transgenic biofortified rice dropped approximately $50 \%$ after 4 months of storage at different storage temperatures. ${ }^{59}$ In order to address the folate-stability problem, the authors followed two strategies. First, a synthetic FBP, based on FBP from bovine milk, was co-expressed with the genes necessary for folate enhancement (GTPCHI and $A D C S) .{ }^{59}$ FBPs are well studied in mammals, but have thus far not been reported in plants. FBPs occur in milk, where they form complexes with folates to protect them from degradation. A second strategy was overexpression of the last enzyme in the folate biosynthesis pathway, folylpolyglutamate synthetase (FPGS). This protein lengthens the glutamate tail of the vitamin, which favors binding to folate-dependent enzymes and promotes cellular retention through its anionic nature. Both strategies appeared to be successful in enhancing folate stability in biofortified rice, and the authors showed that folate levels remained high following long-term storage. ${ }^{59}$ The same principles can be implemented in other folate-biofortified crops. Vitamin-binding proteins are excellent candidates to engineer vitamin stability. Carotenoids are sensitive to oxidation, which compromises the nutritional value of carotenoid-rich food products, such as golden rice, especially during long-term storage. Lipoxygenases (LOX) generate hydroperoxy fatty acids, which are able to co-oxidize and decolorize carotenoids. ${ }^{85,86}$ By aleurone- and endosperm-specific silencing of r9-LOX1 activity through RNA interference, Gayen et al. succeeded in protecting $\beta$-carotene in golden rice from oxidation, hence stabilizing provitamin A content upon storage. ${ }^{87}$ Furthermore, it was demonstrated that the formation of a proper sink structure may provide the ability to promote the accumulation and stability of provitamin A during plant growth and postharvest storage. ${ }^{88}$ Altogether, in order to be successful, biofortification attempts should not only aim at high micronutrient levels but also at a high micronutrient stability, especially when crop products are stored for a long time. The abovementioned examples illustrate that it is possible to engineer vitamin stability. Enhanced vitamin stability can also be obtained through directing vitamin biosynthesis to the accumulation of more stable vitamers or through exploring and manipulating vitamin salvage pathways.

\section{Bioavailability of vitamins in biofortified crop products}

Bioavailability is defined as the fraction of vitamins present in a specific food product or diet that can eventually be used by the human body in metabolic processes. Studying vitamin bioavailability is extremely complex, since it involves many factors that need to be taken into account. These factors include (1) the release of the vitamins from a certain food matrix and (2) uptake by the gastrointestinal tract, which in some cases is accompanied by a conversion of the vitamin to a compound, which can be (3) loaded into the circulation system and (4) transported to different tissues and cells. Bioavailability differs greatly among vitamers. Natural folates, for instance, are less bioavailable than their synthetic counterpart FA, ${ }^{89,90}$ and folate polyglutamates are less bioavailable than folate monoglutamates, since an enzymatic deconjugation reaction is required in the gut mucosa before release into the circulation system. ${ }^{91}$ For vitamin $\mathrm{B}_{6}$, glycosylation of the vitamers lowers bioavailability by $50 \% .{ }^{92}$ Moreover, additional factors present in a diet may influence vitamin bioavailability through interactions among the micronutrients. This was shown for vitamin A, zinc, and iron. ${ }^{93,94}$ Recently, a study revealed that the bioavailability of PACs from papaya 
was higher upon supplementation with iron and zinc after a 5-day PAC-free diet. ${ }^{95}$ In addition, food processing also influences vitamin bioavailability by altering the composition of a food matrix. Pasteurization of orange juice enhanced the bioavailability of $\beta$-cryptoxanthin by 1.8 -fold as compared with fresh oranges, ${ }^{96}$ most likely because more citrus pectin is present in fresh oranges, which negatively affects carotenoid absorption. ${ }^{97,98}$ Vitamin bioavailability is often assessed in murine models, humans, or cell cultures. Kiekens et al. showed via a rat-feeding study that folates from biofortified rice have a high bioavailability, making it a valuable source of dietary folate. ${ }^{99}$ Likewise, a study in humans proved that golden rice is a good source of $\beta$-carotene. ${ }^{100}$ Bioavailability of vitamin $B_{6}$ in biofortified transgenic cassava was proven using human colorectal adenocarcinoma (Caco-2) cells. ${ }^{43}$ Bioavailability is a complex but important aspect that needs to be addressed in biofortification studies. Metabolic engineering can be used to lower factors that negatively influence vitamin bioavailability, such as antinutrients (phytate), in order to enhance compounds that promote bioavailability and/or to aim for the accumulation of vitamers with a high bioavailability.

\section{Multibiofortification through metabolic engineering}

Since staple crops generally have low amounts of several micronutrients, a multibiofortification approach is necessary to eradicate hidden hunger on a large scale. Moreover, this approach appears to be very cost-effective, with a relatively small additional cost compared with the massive increase in health benefits. $^{7}$ A multibiofortification strategy can be interesting not only from a nutritional point of view, but also from a metabolic engineering perspective. As previously mentioned, simultaneous enhancement of several micronutrients can have a positive effect on the absorption (and hence the bioavailability) of these nutrients. Moreover, multibiofortification can positively influence the stability of vitamins (e.g., through their antioxidant properties). Simultaneous biofortification of staple crops with several vitamins or minerals requires a multigene strategy, which is often quite complicated owing to the fact that each (trans)gene needs to be expressed in sufficient amounts, often at the same time and in the same tissues, and this expression needs to remain stable over successive generations. Two homozygous parental lines, each biofortified with one or more different micronutrients, can be crossed to create multibiofortified progeny. Alternatively, retransformation can be used. However, these approaches are labor-intensive and time-consuming, and the introduced traits are prone to segregation. Moreover, this approach requires the use of multiple selectable markers, which is a delicate issue in the deregulation of a crop for consumption. To avoid the use of several selectable markers, co-transformation can be applied. ${ }^{101}$ Ideally, all traits are introduced at a single T-DNA locus, ${ }^{54,59}$ although the limit in the number of (trans)genes at a single T-DNA locus remains an open question.

Until now, most biofortification studies report on the enhancement of a single micronutrient, and only a few examples exist of multibiofortification through metabolic engineering. Naqvi et al. created a multivitamin white corn with enhanced levels of folate (twofold), $\beta$-carotene (169-fold), and ascorbate (sixfold). ${ }^{21}$ In the case of metals, a singlegene approach can result in multiple micronutrient enhancement. Since metal transporters are able to bind different metal cations, engineering metal uptake and transport can result in transgenic crops with enhanced levels of a series of metals. This was clearly illustrated by Lee et al., who showed that the overexpression of OsNAS3 resulted in higher levels of iron, zinc, and copper in transgenic rice. ${ }^{102}$ Similarly, the overexpression of OsNAS2 generated rice with higher seed iron and zinc content. ${ }^{103}$

In the specific case of anemia, a multibiofortification approach is required, since, besides iron deficiency, other factors, such as folate and cobalamin (vitamin $\mathrm{B}_{12}$ ) deficiency, are important contributors. Since cobalamin is only produced by bacteria and archaea, vitamin $B_{12}$ enhancement in crop plants is difficult. ${ }^{5}$ However, the creation of folifer crops - crops with a high folate and iron contentshould be feasible. In this respect, engineering approaches should target GTPCHI and ADCS, both necessary for folate enhancement (in nonrecalcitrant crops), preferably with the overexpression of, for example, FBP to stabilize the accumulated folates upon long-term storage, in combination with genes involved in iron uptake (YSL, IRT, NAS), transport (NAS, YSL) and storage (ferritin). 


\section{Conclusions}

Although biofortification through metabolic engineering is not the ultimate answer to address the malnutrition problem, it surely offers an alternative and/or a complementary strategy to other interventions. In this respect, conventional breeding, where possible, and metabolic engineering should go hand in hand to create multibiofortified crops with health benefits. With our ever-increasing knowledge of micronutrient metabolic pathways and their regulation, metabolic engineering will enable scientists to further improve and fine-tune their biofortification strategies. Several factors, such as micronutrient stability and bioavailability, need to be considered to target specific micronutrient levels in crops in order to meet the different RDAs. Through the use of semistrong promoters driving (trans)gene expression and the selection of engineered lines with moderate but appropriate levels of the target compound(s), the risk of going above the upper limit of the intake of the micronutrient(s), with possible adverse effects on health, can be avoided. With the appearance of new techniques in genome editing, such as TALEN and CRISPR/Cas, the field of pathway engineering will be subjected to a revolution, from which populations suffering from micronutrient deficiencies will surely benefit.

\section{Acknowledgments}

This manuscript was presented at the World Health Organization (WHO)/Food and Agriculture Organization of the United Nations (FAO) technical consultation "Staple crops biofortified with vitamins and minerals: considerations for a public health strategy," convened on April 6-8, 2016, at the Sackler Institute for Nutrition Science, the New York Academy of Sciences in New York, New York. This paper is being published individually but will be consolidated with other manuscripts as a special issue of Annals of the New York Academy of Sciences, the coordinators of which were Drs. Maria Nieves Garcia-Casal and Juan Pablo Peña-Rosas. The special issue is the responsibility of the editorial staff of Annals of the New York Academy of Sciences, who delegated to the coordinators preliminary supervision of both technical conformity to the publishing requirements of Annals of the New York Academy of Sciences and general oversight of the scientific merit of each article. The workshop was supported by the WHO, the FAO, and the Sackler Institute for Nutrition Science at the New York Academy of Sciences. The authors alone are responsible for the views expressed in this paper; they do not necessarily represent the views, decisions, or policies of the institutions with which they are affiliated or the decisions, policies, or views of the WHO. The opinions expressed in this publication are those of the authors and are not attributable to the sponsors, publisher, or editorial staff of Annals of the New York Academy of Sciences. D.B. is indebted to F.W.O. for a postdoctoral fellowship. D.V.D.S. acknowledges support from Ghent University (Bijzonder Onderzoeksfonds, BOF2004/GOA/012 and BOF2009/G0A/004), and the Research Foundation-Flanders (FWO, Projects 3G012609 and 35963). This work was commissioned and financially supported by the Evidence and Programme Guidance Unit, Department of Nutrition for Health and Development of the World Health Organization (WHO), Geneva, Switzerland.

\section{Conflicts of interest}

The authors declare no conflicts of interest.

\section{References}

1. Tulchinsky, T.H. 2010. Micronutrient deficiency conditions: global health issues. Public Health Rev. 32: 243-255.

2. WHO. 2009. Global prevalence of vitamin A deficiency in populations at risk 1995-2005: WHO database on vitamin deficiency. Geneva: World Health Organization.

3. McLaren, D.S., H.A.P.C. Oomen \& H. Escapini. 1966. Ocular manifestations of vitamin-A deficiency in man. Bull. World Health Organ 34: 357-361.

4. Christianson, A., C. Howson \& B. Modell. 2006. March of Dimes global report on birth defects. March of Dimes Birth Defects Foundation, White Plains, New York.

5. Blancquaert, D., H. De Steur, X. Gellynck \& D. Van Der Straeten. 2014. Present and future of folate biofortification of crop plants. J. Exp. Bot. 65: 895-906.

6. Blancquaert, D., S. Storozhenko, K. Loizeau, et al. 2010. Folates and folic acid: from fundamental research toward sustainable health. Crit. Rev. Plant Sci. 29: 14-35.

7. De Steur, H., D. Blancquaert, S. Strobbe, et al. 2015. Status and market potential of transgenic biofortified crops. Nat. Biotechnol. 33: 25-29.

8. Farré, G., D. Blancquaert, T. Capell, et al. 2014. Engineering complex metabolic pathways in plants. Annu. Rev. Plant Biol. 65: 187-223.

9. Ross, S.A., P.J. McCaffery, U.C. Drager \& L.M. De Luca. 2000. Retinoids in embryonal development. Physiol. Rev. 80: 1021-1054.

10. West, K.P., Jr. \& I. Darnton-Hill. 2001. Vitamin A deficiency. In Nutrition and Health in Developing Countries. 
R.D. Semba \& M.W. Bloem, Eds.: 267-306. Totowa, NJ: Humana Press.

11. Misawa, N., M.R. Truesdale, G. Sandmann, et al. 1994. Expression of a tomato cDNA coding for phytoene synthase in Escherichia coli, phytoene formation in vivo and in vitro, and functional analysis of the various truncated gene products. J. Biochem. 116: 980-985.

12. Chappell, J. 1995. Biochemistry and molecular biology of the isoprenoid biosynthetic pathway in plants. Ann. Rev. Plant Physiol. Plant Mol. Biol. 46: 521-547.

13. Isaacson, T., I. Ohad, P. Beyer \& J. Hirschberg. 2004. Analysis in vitro of the enzyme CRTISO establishes a poly-ciscarotenoid biosynthesis pathway in plants. Plant Physiol. 136: 4246-4255.

14. Breitenbach, J. \& G. Sandmann. 2005. $\zeta$-Carotene cis isomers as products and substrates in the plant poly-cis carotenoid biosynthetic pathway to lycopene. Planta 220: 785-793.

15. Li, Q., G. Farre, S. Naqvi, et al. 2010. Cloning and functional characterization of the maize carotenoid isomerase and $\beta$-carotene hydroxylase genes and their regulation during endosperm maturation. Transgenic Res. 19: 1053-1068.

16. Farré, G., G. Sanahuja, S. Naqvi, et al. 2010. Travel advice on the road to carotenoids in plants. Plant Sci. 179: 28-48.

17. Zhu, C., C. Bai, G. Sanahuja, et al. 2010. The regulation of carotenoid pigmentation in flowers. Arch. Biochem. Biophys. 504: 132-141.

18. Tian, L., M. Magallanes-Lundback, V. Musetti \& D. DellaPenna. 2003. Functional analysis of beta- and epsilonring carotenoid hydroxylases in Arabidopsis. Plant Cell 5: $1320-1332$.

19. Ye, X., S. Al-Babili, A. Kloti, et al. 2000. Engineering the provitamin A ( $\beta$-carotene) biosynthetic pathway into (carotenoid-free) rice endosperm. Science 287: 303-305.

20. Paine, J.A., C.A. Shipton, S. Chaggar, et al. 2005. Improving the nutritional value of golden rice through increased provitamin A content. Nat. Biotechnol. 23: 482-487.

21. Naqvi, S., C. Zhu, G. Farre, et al. 2009. Transgenic multivitamin corn through biofortification of endosperm with three vitamins representing three distinct metabolic pathways. Proc. Natl. Acad. Sci. U.S.A. 106: 7762-7767.

22. Cong, L., C. Wang, L. Chen, et al. 2009. Expression of phytoene synthasel and carotene desaturase crtl genes result in an increase in the total carotenoids content in trangenic elite wheat (Triticum aestivum L.). J. Agric. Food Chem. 57: $8652-8660$

23. Sayre, R., J.R. Beeching, E.B. Cahoon, et al. 2011. The BioCassava-Plus Program: biofortification of cassava for subSaharan Africa. Annu. Rev. Plant Biol. 62: 251-272.

24. Lipkie, T.E., F.F. De Moura, Z.Y. Zhao, et al. 2013. Bioaccessibility of carotenoids from transgenic provitamin A biofortified sorghum. J. Agric. Food Chem. 61: 5764-5771.

25. Diretto, G., S. Al-Babili, R. Tavazza, et al. 2007. Metabolic engineering of potato carotenoid content through tuberspecific overexpression of a bacterial mini-pathway. PLoS ONE 2: e350.

26. Ricciarelli, R., J.M. Zingg \& A. Azzi. 2001. Vitamin E: protective role of a Janus molecule. FASEB J. 15: 2314-2325.

27. Gilliland, L.U., M. Magallanes-Lundback, C. Hemming, et al. 2006. Genetic basis for natural variation in seed vita- min E levels in Arabidopsis thaliana. Proc. Natl. Acad. Sci. U.S.A. 103: 18834-18841.

28. Karunanandaa, B., Q. Qi, M. Hao, et al. 2005. Metabolically engineered oilseed crops with enhanced seed tocopherol. Metab. Eng. 7: 384-400.

29. Farré, G., D. Sudhakar, S. Naqvi, et al. 2012. Transgenic rice grains expressing a heterologous $\rho$-hydroxyphenylpyruvate dioxygenase shift tocopherol synthesis from the $\gamma$ to the $\alpha$ isoform without increasing absolute tocopherol levels. Transgenic Res. 21:1093-1097.

30. Cahoon, E.B., S.E. Hall, K.G. Ripp, et al. 2003. Metabolic redesign of vitamin E biosynthesis in plants for tocotrienol production and increased antioxidant content. Nat. Biotechnol. 21: 1082-1087.

31. Naqvi, S., G. Farré, C. Zhu, et al. 2011. Simultaneous expression of Arabidopsis $\rho$-hydroxyphenylpyruvate dioxygenase and MPBQ methyltransferase in transgenic corn kernels triples the tocopherol content. Transgenic Res. 20: 177-181.

32. Zhang, G.Y., R.R. Liu, G. Xu, et al. 2013. Increased $\alpha-$ tocotrienol content in seeds of transgenic rice overexpressing Arabidopsis $\gamma$-tocopherol methyltransferase. Transgenic Res. 22: 89-99.

33. Hellmann, H. \& S. Mooney. 2010. Vitamin $\mathrm{B}_{6}$ : a molecule for human health? Molecules 15: 442-459.

34. Tanaka, T., Y. Tateno \& T. Gojobori. 2005. Evolution of vitamin $\mathrm{B}_{6}$ (pyridoxine) metabolism by gain and loss of genes. Mol. Biol. Evol. 22: 243-250.

35. Tambasco-Studart, M., O. Titiz, T. Raschle, et al. 2005. Vitamin $\mathrm{B}_{6}$ biosynthesis in higher plants. Proc. Natl. Acad. Sci. U.S.A. 102: 13687-13692.

36. Ehrenshaft, M., P. Bilski, M. Li, C. Chignell, et al. 1999. A highly conserved sequence is a novel gene involved in de novo vitamin $\mathrm{B}_{6}$ biosynthesis. Proc. Natl. Acad. Sci. U.S.A. 96: $9374-9378$.

37. Mittenhuber, G. 2001. Phylogenetic analyses and comparative genomics of vitamin $\mathrm{B}_{6}$ (pyridoxine) and pyridoxal phosphate biosynthesis path ways. J. Mol. Microbiol. Biotechnol. 3: 1-20.

38. Ouyang, S., W. Zhu, J. Hamilton, et al. 2007. The TIGR rice genome annotation resource: improvements and new features. Nucleic Acids Res. 35: 883-887.

39. Prochnik, S., P.R. Marri, B. Desany, et al. 2012. The cassava genome: current progress, future directions. Trop. Plant Biol. 5: 88-94.

40. Herrero, S. \& M.E. Daub. 2007. Genetic manipulation of vitamin $\mathrm{B}_{6}$ biosynthesis in tobacco and fungi uncovers limitations to up-regulation of the pathway. Plant Sci. 172: 609-620.

41. Chen, H. \& L.M. Xiong. 2009. Enhancement of vitamin $\mathrm{B}_{6}$ levels in seeds through metabolic engineering. Plant Biotechnol. J. 7: 673-681.

42. Raschke, M., S. Boycheva, M. Crèvecoeur, et al. 2011. Enhanced levels of vitamin $\mathrm{B}_{6}$ increase aerial organ size and positively affects tress tolerance in Arabidopsis. Plant J. 66: 414-432.

43. Li, K.T., M. Moulin, N. Mangel, et al. 2015. Increased bioavailable vitamin B-6 in field-grown transgenic cassava for dietary sufficiency. Nat. Biotechnol. 33: 1029-1032.

44. Ravanel, S., R. Douce \& F. Rébeillé. 2011. Metabolism of folates in plants. Adv. Bot. Res. 59: 67-106. 
45. Geisel, J. 2003. Folic acid and neural tube defects in pregnancy: a review. J. Perinat. Neonat. Nurs. 17: 268-279.

46. Li, G.M., S.R. Presnell \& L.Y. Gu. 2003. Folate deficiency, mismatch repair-dependent apoptosis, and human disease. J. Nutr. Biochem. 14: 568-575.

47. Scott, J.M. \& D.G. Weir. 1996. Homocysteine and cardiovascular disease. Q. J. Med. 89: 561-563.

48. Stanger, O. 2004. The potential role of homocysteine in percutaneous coronary interventions (PCI): review of current evidence and plausibility of action. Cell. Mol. Biol. 50: 953-988.

49. Endres, M., M. Ahmadi, I. Kruman, et al. 2005. Folate deficiency increases postischemic brain injury. Stroke 36: 321-325.

50. Seshadri, S., A. Beiser, J. Selhub, et al. 2002. Plasma homocysteine as a risk factor for dementia and Alzheimer's disease. N. Engl. J. Med. 346: 476-483.

51. Choi, S.W. \& S. Friso. 2005. Interactions between folate and aging for carcinogenesis. Clin. Chem. Lab. Med. 43: 1151-1157.

52. Hossain, T., I. Rosenberg, J. Selhub, et al. 2004. Enhancement of folate in plants through metabolic engineering. Proc. Natl. Acad. Sci. U.S.A. 101: 5158-5163.

53. Blancquaert, D., S. Storozhenko, J. Van Daele, et al. 2013. Enhancing pterin and para-aminobenzoate content is not sufficient to successfully biofortify potato tubers and Arabidopsis thaliana plants with folate. J. Exp. Bot. 64: 38993909.

54. Storozhenko, S., V. De Brouwer, M. Volckaert, et al. 2007. Folate fortification of rice by metabolic engineering. Nat. Biotechnol. 25: 1277-1279.

55. Dong, W., Z.-J. Cheng, C.-L. Lei, et al. 2014. Overexpression of folate biosynthesis genes in rice (Oryza sativa L.) and evaluation of their impact on seed folate content. Plant Food. Hum. Nutr. 69: 379-385.

56. Diaz de la Garza, R., E.P. Quinlivan, S.M. Klaus, et al. 2004. Folate biofortification in tomatoes by engineering the pteridines branch of folate synthesis. Proc. Natl. Acad. Sci. U.S.A. 101: 13720-13725.

57. Diaz de la Garza, R.I., J.F. Gregory III \& A.D. Hanson. 2007. Folate biofortification of tomato fruit. Proc. Natl. Acad. Sci. U.S.A. 104: 4218-4222.

58. Nunes, A.C.S., D.C. Kalkmann \& F.J.L. Aragão. 2009. Folate biofortification of lettuce by expression of a codon optimized chicken GTP cyclohydrolase I gene. Transgenic Res. 18: 661-667.

59. Blancquaert, D., J. Van Daele, S. Strobbe, et al. 2015. Improving folate (vitamin $\mathrm{B}_{9}$ ) stability in biofortified rice through metabolic engineering. Nat. Biotechnol. 33: 10761078.

60. WHO. 2002. The world health report. Geneva: World Health Organization.

61. Wu, H., L. Li, J. Du, et al. 2005. Molecular and biochemical characterization of the $\mathrm{Fe}$ (III) chelate reductase gene family in Arabidopsis thaliana. Plant Cell Physiol. 46: 15051514.

62. Vasconcelos, M., V. Musetti, C.-M. Li, et al. 2004. Functional analysis of transgenic rice (Oryza sativa L.) transformed with an Arabidopsis thaliana ferric reductase (AtFRO2). Soil Sci. Plant Nutr. 50: 1151-1157.
63. Vasconcelos, M.W., T.E. Clemente \& M.A. Grusak. 2014. Evaluation of constitutive iron reductase (AtFRO2) expression on mineral accumulation and distribution in soybean (Glycine max L.). Front. Plant Sci. 5: 112.

64. Stephan, U.W. \& G. Scholz. 1993. Nicotianamine: a mediator of transport of iron and heavy metals in the phloem? Physiol. Plant. 88: 522-529.

65. Morrissey, J. \& M.L. Guerinot. 2009. Iron uptake and transport in plants: the good, the bad, and the ionome. Chem. Rev. 109: 4553-4567.

66. Curie, C., G. Cassin, D. Couch, et al. 2009. Metal movement within the plant: contribution of nicotianamine and yellow stripe 1-like transporters. Ann. Bot. 103: 1-11.

67. Ravet, K., B. Touraine, J. Boucherez, et al. 2009. Ferritins control interaction between iron homeostasis and oxidative stress in Arabidopsis. Plant J. 57: 400-412.

68. Raboy, V., P.F. Gerbasi, K.A. Young, et al. 2000. Origin and seed phenotype of maize low phytic acid 1-1 and low phytic acid 2-1. Plant Physiol. 124: 355-368.

69. Cromwell, G.L., T.S. Stahly, R.D. Coffey, et al. 1993. Efficacy of phytase in improving the bioavailability of phosphorus in soybean-meal and corn-soybean meal diets for pigs. J. Anim. Sci. 71: 1831-1840.

70. Brinch-Pedersen, H., L.D. Sorensen \& P.B. Holm. 2002. Engineering crop plants: getting a handle on phosphate. Trends Plant Sci. 7: 118-125.

71. Kuwano, M., A. Ohyama, Y. Tanaka, et al. 2006. Molecular breeding for transgenic rice with low-phytic-acid phenotype through manipulating myo-inositol 3-phosphate synthase gene. Mol. Breed. 18: 263-272.

72. Ali, N., S. Paul, D. Gayen, et al. 2013. Development of low phytate rice by RNAi mediated seed-specific silencing of inositol 1,3,4,5,6-pentakisphosphate 2-kinase gene (IPK1). PLoS One 8: e68161.

73. Shi, J.R., H.Y. Wang, K. Schellin, et al. 2007. Embryospecific silencing of a transporter reduces phytic acid content of maize and soybean seeds. Nat. Biotechnol. 25: 930937.

74. Raboy, V. 2007. The ABCs of low-phytate crops. Nat. Biotechnol. 25: 874-875.

75. Zielinska-Dawidziak, M. 2015. Plant ferritin-a source of iron to prevent its deficiency. Nutrients 7: 1184-1201.

76. Ravet, K., B. Touraine, S.A. Kim, et al. 2009b. Posttranslational regulation of AtFER2 ferritin in response to intracellular iron trafficking during fruit development in Arabidopsis. Mol. Plant 2: 1095-1106.

77. Masuda, H., Y. Ishimaru, M.S. Aung, et al. 2012. Iron biofortification in rice by the introduction of multiple genes involved in iron nutrition. Sci. Rep. 2: 543.

78. Fitzpatrick, T.B., G.J. Basset, P. Borel, et al. 2012. Vitamin deficiencies in humans: can plant science help? Plant Cell 24: 395-414.

79. Rébeillé, F., S. Ravanel, S. Jabrin, et al. 2006. Folates in plants: biosynthesis, distribution and enhancement. Physiol. Plant. 126: 330-342.

80. Cortes, M., A. Chiralt \& H. Suarez. 2009. Influence of storage conditions on freeze-dried apple fortified with vitamin E. Vitae-Columbia 16: 31-41.

81. Ordonez-Santos, L.E. \& A. Vasquez-Riascos. 2010. Effect of processing and storage time on the vitamin $\mathrm{C}$ and lycopene 
contents of néctar of pink guava (Psidium guajava L.). Arch. Latinoam. Nutr. 60: 280-284.

82. Mugode, L., B. Ha, A. Kaunda, et al. 2014. Carotenoid retention of biofortified provitamin A maize (Zea mays L.) after Zambian traditional methods of milling, cooking and storage. J. Agric. Food Chem. 62: 6317-6325.

83. Choi, S., H. Jun, J. Bang, et al. 2015. Behaviour of Aspergillus flavus and Fusarium graminearum on rice as affected by degree of milling, temperature, and relative humidity during storage. Food Microbiol. 46: 307-313.

84. Mejia, D. 2003. Maize: post-harvest operations. Rome: FAO.

85. Casey, R. 1997. Lipoxygenases and breadmaking. In Proceedings of the First European Symposium on Enzymes and Grain Processing. S.A.G.F. Angelino, R.J. Hamer, W. van Hartingsfeld, F. Heidekamp \& J.P. van der Lugt, Eds.: 188194. Noordwijkerhout, the Netherlands.

86. Wu, Z., D.S. Robinson, R.K. Hughes, et al. 1999. Cooxidation of beta-carotene catalyzed by soybean and recombinant pea lipoxygenases. J. Agric. Food Chem. 47: 4899-490.

87. Gayen, D., N. Ali, S.N. Sarkar, et al. 2015. Down-regulation of lipoxygenase gene reduces degradation of carotenoids of golden rice during storage. Planta 242: 353-363.

88. Li, L., Y. Yang, Q. Xu, et al. 2012. The Or gene enhances carotenoid accumulation and stability during post-harvest storage of potato tubers. Mol. Plant 5: 339-352.

89. Food and Drug Administration. 1996. Food standards: amendments of standards of identity for enriched grain products to require addition of folic acid: final rule (21 CFR parts 136, 137, 139). Fed. Reg. 61: 8781-8807.

90. Bailey, L.B. 2004. Folate and vitamin $B_{12}$ recommended intakes and status in the United States. Nutr. Rev. 62: S14S20.

91. Melse-Boonstra, A., C.E. West, M.B. Katan, et al. 2004. Bioavailability of heptaglutamyl relative to monoglutamyl folic acid in healthy adults. Am. J. Clin. Nutr. 79: 424-429.

92. Gregory, J.F., III. 2012. Accounting for differences in the bioactivity and bioavailability of vitamers. Food Nutr. Res. 56: 5809 .
93. Dijkhuizen, M.A., F.T. Wieringa, C.E. West \& Muhilal. 2004. Zinc plus $\beta$-carotene supplementation of pregnant women is superior to $\beta$-carotene supplementation alone in improving vitamin A status in both mothers and infants. Am. J. Clin. Nutr. 80: 1299-1307.

94. Bloem, M.W. 1995. Interdependence of vitamin A and iron: an important association for programs of anaemia control. Proc. Nutr. Soc. 54: 501-508.

95. Kana-Sop, M.M., I. Gouado, M.B. Achu, et al. 2015. The influence of rion and zinc supplementation on the bioavailability of provitamin A carotenoids from papaya following consumption of a vitamin A-deficient diet. J. Nutr. Sci. Vitaminol. 61: 205-214.

96. Aschoff, J.K., C.L. Rolke, N. Breusing, et al. 2015. Bioavailability of beta-croptoxanthin is greater from pasteurized orange juice than from fresh oranges-a randomized crossover study. Mol. Nutr. Food Res. 59: 1896-1904.

97. Rock, C.L. \& M.E. Swendseid. 1992. Plasma beta-carotene response in humans after meals supplemented with dietary pectin. Am. J. Clin. Nutr. 55: 96-99.

98. Riedl, J., J. Linseisen, J. Hoffmann \& G. Wolfram. 1999. Some dietary fibers reduce the absorption of carotenoids in women. J. Nutr. 129: 2170-2176.

99. Kiekens, F., D. Blancquaert, L. Devisscher, et al. 2015. Folates from metabolically engineered rice: a long-term study in rats. Mol. Nutr. Food Res. 59: 490-500.

100. Tang, G.W., J. Qin, G.G. Dolnikowski, et al. 2009. Golden rice is an effective source of vitamin A. Am. J. Clin. Nutr. 89: $1776-1783$.

101. Halpin, C. 2005. Gene stacking in transgenic plants-the challenge for the 21st century plant biotechnology. Plant Biotechnol. J. 3: 141-155.

102. Lee, S., U.S. Jeon, S.J. Lee, et al. 2009. Iron fortification of rice seeds through activation of the nicotianamine synthase gene. Proc. Natl. Acad. Sci. U.S.A. 106: 2201422019.

103. Johnson, A.A.T., B. Kyriacou, D.L. Callahan, et al. 2011. Constitutive overexpression of the OsNAS gene family reveals single-gene strategies for effective iron- and zincbiofortification of rice endosperm. PLoS One 6: e24476. 